\title{
Regulation of Ecto-5'-Nucleotidase by Docosahexaenoic Acid in Human Endothelial Cells
}

\author{
Vu Thi Thom Martina Wendel Andreas Deussen \\ Department of Physiology, Medical Faculty Carl Gustav Carus, TU Dresden, Germany
}

\author{
Key Words \\ Ecto-ATPDase $・$ Ecto-5'-nucleotidase $・$ Docosahexaenoic acid $\bullet$ Endothelial cells
}

\begin{abstract}
Background/Aims: Modulation of extracellular adenine nucleotide and adenosine concentrations is one potential mechanism by which docosahexaenoic acid (DHA) may exert beneficial effects in critically ill patients. This study assessed DHA effects on extracellular adenine purines. Methods: Experiments used human pulmonary endothelial cells (HPMEC) and umbilical vein endothelial cells (HUVEC) treated with DHA (48 h). mRNA level (real-time $P C R$ ), expression (western blot, flow cytometry) and activities (hydrolysis of etheno $(\varepsilon)$-purines and fluorescence HPLC) of CD73 (ecto-5'-nucleotidase) and CD39 (ecto-NTPDase-1) were quantified. Results: DHA elevated total CD73 membrane protein expression concentrationdependently but CD73 mRNA level did not change. Increased expression was paralleled by increased enzyme activity. Effects observed on membrane level were reversed in intact cells, in which $\varepsilon$-AMP hydrolysis decreased after DHA. In intact endothelial cells ATP release was enhanced and CD39 activity blunted following DHA treatment. Hence, extracellular ATP and ADP concentrations increased and this inhibited $\varepsilon$-AMP hydrolysis. Conclusion: In human endothelial cells DHA caused 1) up-regulation of CD73 protein content and increased AMP hydrolysis at the cell membrane level, 2) increased cellular ATP release, and 3) decreased extracellular ATP/ADP hydrolysis. Thus, reorganization of the extracellular adenine-nucleotideadenosine axis in response to DHA resulted in an increased extracellular ATP/adenosine ratio.
\end{abstract}

Copyright @ 2013 S. Karger AG, Basel

\section{Introduction}

Modulation of extracellular adenine nucleotide and adenosine concentrations has been proposed to cause tissue and organ protective effects. In particular modulation of ecto-5' nucleotidase (CD73), the rate controlling enzyme in the stepwise degradation of extracellular

Dr. Andreas Deussen

Professor and Chair
Department of Physiology, Medical Faculty Carl Gustav Carus, TU Dresden

Fetscherstr. 74, D-01307 Dresden (Germany)

Tel. +49 351 4586030, Fax +49 351 4586301, E-Mail Andreas.Deussen@tu-dresden.de 
ATP to adenosine has attracted much interest in the past. However, a conclusive model has not yet been established. Protective effects observed after blunting of CD73 activity include reduced airway responsiveness after allergen exposure [1], reduced hepatic fibrosis generation [2], and decreased endothelial layer permeability [3]. In contrast, augmented tissue injury after blunting of adenosine production by CD73 has been reported under conditions of acute lung injury induced by mechanical ventilation [4], hypoxic lung injury [5] and hypoxia induced hyperpermeability of intestinal mucosa [6].

Previous studies indicated increased CD73 expression and CD73 enzymatic activity of endothelial cell membrane preparations after exposure to docosahexaenoic acid (DHA), a polyunsaturated fatty acid (PUFA) [7]. This is of potential clinical relevance, because PUFA treatment of critically ill patients with systemic inflammatory response syndrome and acute respiratory distress syndrome resulted in reduced neutrophil recruitment and improved lung gas exchange associated with reduced mortality rates and shorter time of recovery [8, 9]. In isolated human endothelial cell culture DHA attenuated monocytic cell adhesion [10]. Because adenosine production via CD73 has been demonstrated to ameliorate inflammatory vascular reactions $[4,5,11]$, part of the effects of DHA have been attributed to the upregulation of CD73 activity [7]. However, as outlined above, the potential effects of CD73 inhibition remain controversial [1-6].

A major limitation of previous studies addressing a possible causal relationship between DHA treatment and CD73 results from the exclusive use of isolated cell membrane preparations [7]. Although this model permits to investigate modulating effects of DHA on enzyme expression and activity, it does not give insight into influences or kinetic aspects that may be relevant at the whole cell or tissue level. Endothelial cells are known to release adenine nucleotides in response to a variety of stimuli [12-14]. In addition to CD73, ectoATPase and ecto-ADPase (NTPDase-1 or CD39) activity may be changed by DHA treatment. This may consecutively affect CD73 activity, because ADP and ATP are powerful modulators of CD73 activity $[15,16]$. Thus, it is presently unclear, whether intact endothelial cell CD73 activity is increased as suggested from cell membrane preparations or decreased in response to DHA treatment due to more complex regulatory events on the cellular level. Furthermore, alkaline phosphatase, which hydrolyses extracellular adenine nucleotides in parallel to CD73, should be included in a comprehensive analysis of adenine nucleotide metabolism [17].

The present study was designed to comprehensively test the effects of DHA on CD73 and alkaline phosphatase activities of human endothelial cells. Experiments performed in membrane preparations showed the effect described in literature, namely the up-regulation of CD73 total protein, protein surface content and enzyme activity reflected by the $\varepsilon$-AMP hydrolysis rate. However, in intact endothelial cells DHA reduced $\varepsilon$-AMP to $\varepsilon$-Ado conversion. This effect was caused by increased ATP release and inhibited CD39 (ecto-ATPDase) activity in response to DHA. In contrast to CD73, ecto-alkaline phosphatase activity was unimportant. Thus, DHA augments extracellular availability of adenine nucleotides and depresses extracellular adenosine production in human vascular endothelial cells.

\section{Materials and Methods}

The study conforms to the principles of the Declaration of Helsinki. It was conducted under permission EZ 203112005 of the local authorities.

Cell culture

Human umbilical vein endothelial cells (HUVEC) were isolated under sterile conditions within a time window of $24 \mathrm{~h}$ after delivery following standard procedures. Briefly, the cords were incubated with $0.05 \%$ collagenase solution (PAA, Coelbe, Germany) in PBS (Invitrogen, Darmstadt, Germany) for 11 min at $37^{\circ} \mathrm{C}$. After harvesting of endothelial cells they were re-suspended in M199 medium (GIBCO, Darmstadt, Germany) containing $10 \%$ FCS and grown on $0.5 \%$ gelatine type A (Sigma, München, Germany) coated T25 dishes in an incubator $\left(5 \% \mathrm{CO}_{2}\right.$ ). Human Pulmonary Microvascular Endothelial Cells (HPMEC-ST1.6R) obtained from 
Thom/Wendel/Deussen: DHA and Extracellular Adenine Nucleotide Metabolism

Prof. Dr. med. C. J. Kirkpatrick (Institute of Pathology, Johannes Gutenberg University Mainz, Germany) [18] were grown similar to HUVEC. For augmentation confluent HUVEC and sub-confluent HPMEC were split in $3.5 \mathrm{~cm}$ dishes. Experiments were performed in supplemented ECGM-2 (PromoCell, Heidelberg, Germany) medium.

\section{DHA treatment}

Approximately 50.000 cells were seeded on a $3.5 \mathrm{~cm}$ diameter cell culture dish supplemented with culture medium. When the cells were 80-90 \% confluent, the medium was renewed and supplemented with antioxidants including $50 \mu \mathrm{mol} / \mathrm{l}$ of alpha-tocopherol (Sigma) and $300 \mu \mathrm{mol} / \mathrm{l}$ of ascorbic acid (Pharmacy, University Hospital Dresden, Germany). Within the next 24 h DHA (IBL Hamburg, Germany) taken from a stock solution in ethanol (DHA concentration $761 \mathrm{mmol} / \mathrm{l}$ ) was diluted freshly for experiments in culture medium supplemented with antioxidants and applied to cells $(24,48,72 \mathrm{~h})$. Final concentrations were 0,25 , $50 \mu \mathrm{mol} / \mathrm{l}$ (HUVEC) and 0, 25, 50, $100 \mu \mathrm{mol} / \mathrm{l}$ (HPMEC). For all experiments cells were no more than 1 week old first passage HUVEC or less than the fiftieth passage HPMEC. DHA was protected from access of oxygen and DHA stock solutions were generally discarded after 3-4 months.

\section{Flow cytometry}

Flow cytometry was used to assess CD73 protein surface expression. Briefly, cultured HUVEC and HPMEC were trypsinized for 0.5 to $1 \mathrm{~min}$. After reaction of trypsin was stopped with serum, cells were harvested and cell pellets washed with PBS, followed by fixation with $2 \%$ paraformaldehyde (Sigma) for 10 min on ice. After a blocking step with $10 \%$ normal serum (PAA), the cells were incubated with primary antibodies (mouse anti-CD73 IE9, Santa Cruz) at $4^{\circ} \mathrm{C}$ overnight. Primary antibodies were detected with a respective secondary antibody (anti-mouse Alexa 488, Santa Cruz). Measurements were performed in a BD FACS Calibur ${ }^{\mathrm{TM}}$. The results were quantified by Win MDI 2.9 software with gating procedure.

\section{Protein determination and western blot}

To detect CD73, CD39, caveolin-1 and annexin-2 expression, samples were collected directly after enzymatic assay by adding Tris/ $\mathrm{HCl}$ pH 6.9 buffer. Samples were then immediately stored at $-20^{\circ} \mathrm{C}$. Protein concentration in the samples was determined by both the black amido protein assay and the rotinanoquant assay (Carl Roth, Germany). Protein expression was quantified with western blot. Briefly, equal amounts (20-30 $\mu \mathrm{g}$ ) of protein were loaded on 4 to $12 \%$ polyacrylamide gels (Bio Rad Laboratories $\mathrm{GmbH}$, Germany). After electrophoretic separation the proteins were transferred onto nitrocellulose membrane (Amersham Biosciences) following standard procedures. The membrane was then blocked with $5 \%$ skim milk. The membranes were incubated in respective primary antibodies to detect CD73 (1:200, Santa Cruz Biotechnology), CD39 (1:200, Santa Cruz), Cav-1 (1:5000, rabbit, BD Biosciences), annexin-2 (1:2000, rabbit, Santa Cruz). Amounts of target proteins was normalised to $\alpha$-tubulin and $\beta$-actin (both 1:1000, Sigma). Secondary antibodies used were donkey anti-goat (1:2000, Dianova, Hamburg, Germany), goat antimouse (1:1000, Dianova) and goat anti-rabbit (1:1000, Dianova). Protein bands were quantified using an Alias 3000 Reader. Blot images were quantified densitometrically using Image J software.

\section{Real time Polymerase Chain Reaction}

From HPMEC incubated with DHA for 36 and 48 hours RNA was isolated using the RNeasy Mini Kit (Qiagen NV, The Netherlands). RT-PCR was performed by standard procedures using the following primers: 1) ecto-5'-nucleotidase/CD73: sense 5' GAT CGA GCC ACT CCT CAA A 3'; antisense 5' GCC CAT CAT CAG AAG TGA C 3'; 2) GAPDH: sense 5'CAA AGT TGT CAT GGATGA CC3'; antisense 5'CCA TGG AGA AGG CTG GGG3'. Thermal cycling conditions were done as follows: first step, $94^{\circ} \mathrm{C}$ for $15 \mathrm{~min}$; the ensuing 40 cycles were $95^{\circ} \mathrm{C}$ for $20 \mathrm{~s}, 58^{\circ} \mathrm{C}$ for $30 \mathrm{~s}$ and $72^{\circ} \mathrm{C}$ for $30 \mathrm{~s}$.

\section{HPLC assessment of adenine nucleotides and adenosine}

Measurement of etheno( $(\varepsilon)$-labelled adenine nucleotides and adenosine. CD73 activity was determined by measuring the conversion of the substrate $\varepsilon$-AMP (Sigma) to $\varepsilon$-adenosine ( $\varepsilon$-Ado) using fluorescence HPLC as described before [19]. The kinetic of $\varepsilon$-AMP conversion to $\varepsilon$-Ado was plotted and the time point of $50 \%$ conversion of $\varepsilon$-AMP to $\varepsilon$-Ado was expressed as $t_{50}$ value. For statistical analysis, $t_{50}$ values in the treatment groups were normalized to their respective controls treated in parallel. CD39 activity was 
measured similarly to CD73 activity, however, $\varepsilon$-ATP (Molecular Probes, Darmstadt, Germany) and $\varepsilon$-ADP (Sigma), rather than $\varepsilon$-AMP, were added as substrates.

Measurement of native adenine nucleotides and adenosine. HUVEC and HPMEC cultured and incubated with DHA in serum culture medium ( $48 \mathrm{~h}$ ) were washed once with PBS and $2 \mathrm{ml}$ incubation buffer were added. Supernatant $(500 \mu \mathrm{l})$ was collected after 0,16 and $32 \mathrm{~min}$ of incubation, respectively, immediately frozen in liquid nitrogen and stored at $-80^{\circ} \mathrm{C}$ until freeze-drying. The residue was redissolved in $143 \mu \mathrm{l}$ of ultra pure water. For quantification of native adenine compounds a derivatization procedure to etheno $(\varepsilon)$ compounds was used as described [19]. HPLC measurements were performed as reported [20]. Identification and quantification of $\varepsilon$-adenosine and $\varepsilon$-adenine nucleotides were done using external standards.

\section{ATP measurement by luminometry}

Cellular native ATP release was measured using an ATP-time stable determination kit (Proteinkinase, Biaffin GmbH \& Co KG, Kassel, Germany) which is based on luciferase activity. The fluorescence signal was measured in a luminometer as a function of time and the ATP concentration quantified from the area under the curve using external ATP standards (Sigma) as reference. The ATP release of DHA treated cells was normalized to that of control experiments performed in parallel.

\section{Statistical analysis}

Statistical analysis was performed using SPSS for Windows version 15.0. Data were expressed as mean \pm SE. Differences of mean values between two groups were analysed by Student's $t$ test. When more than two groups were compared, statistical analysis was performed by a one-way ANOVA followed by post hoc test. A p-value $<0.05$ was considered to indicate a statistically significant difference.

\section{Results}

DHA up-regulates CD73 protein level

To determine the effects of DHA on CD73 protein level we quantified CD73 surface expression by flow cytometry for both HPMEC and HUVEC (Fig. 1A). After $48 \mathrm{~h}$ DHA treatment CD73 protein surface content increased dose-dependently in both cell types. At $50 \mu \mathrm{mol} / \mathrm{l}$ DHA the increase of CD73 expression was $28 \pm 7 \%$ (HPMEC) and $23 \pm 6 \%$ (HUVEC) as compared to respective controls $(\mathrm{p}<0.05, \mathrm{n}=6-8)$. A representative histogram of FL1 fluorescence labelling of CD73 is provided in Fig. 1B. DHA did not have any cytotoxic effect in HUVEC and HPMEC as revealed by MTT assay and LDH release (data not shown). The enhanced CD73 cell surface content indicated by flow cytometry was paralleled by a similar increase in whole cell CD73 protein level. Cellular CD73 protein content increased by $41 \pm 11 \%$ and $48 \pm 10 \%$ in HPMEC and HUVEC, respectively, in response to $50 \mu \mathrm{mol} / \mathrm{l}$ DHA for 48 hours ( $p<0.05, n=5-6$, Fig. 1C and 1D).

Recently, DHA as well as some other fatty acids e.g eicosapentaenoic acid and arachidonic acid have been described as potential ligands of the PPAR family including PPAR- $\gamma$ [21]. Because DHA had no effect on CD73 mRNA level (Fig. 1E), we evaluated whether DHA via the PPAR- $\gamma$ pathway may directly regulate some unknown genes which play a role in CD73 expression and enzyme activity. However, application of GW9962 for $72 \mathrm{~h}$ had no effect on both CD73 activity (data not shown) and CD73 protein level (Fig. 1F). This suggests that DHA regulated CD73 independent of the PPAR- $\gamma$ pathway.

\section{Modulation of lipid raft compositions on CD73 enzymatic activity}

As CD73 is known to be located in lipid rafts one may assume that modulation of the lipid membrane composition such as presence of cholesterol or caveolin-1 (cav-1) may modify CD73 activity or CD73 protein expression. We performed experiments in which the cholesterol content in the lipid raft was depleted by filipin (Fig. 2A) and beta-methylcyclodextrin (MCD) treatment (Fig. 2B). In further experiments administration of MCD-cholesterol complex was used to enrich the membrane cholesterol content (Fig. 2B). However, both displacement and enrichment of cholesterol level had no effect on CD73 activity. In agreement with 
Fig. 1. Effects of DHA on CD73 protein expression. A: Dose-dependent regulation of CD73 surface expression in response to $48 \mathrm{~h}$ DHA treatment in HPMEC and HUVEC as determined by flow cytometry. Values are given as percent change of the mean fluorescence intensity FL-1 relative to the untreated control ( $n \geq 6$ experiments). B: Representative histogram of FL-1 fluorescence intensity in HPMEC labelled with mouse anti-human CD73 antibody, IE9 and antimouse alexa 488. C\&D: CD73 protein expression in HPMEC and HUVEC determined by western blotting after 48 h DHA treatment (C quantitative data, D representative western blot). Densitometric measurements were normalized to $\alpha$-tubulin expression and values are given as percent change compared to untreated controls $(n=5-6),{ }^{*} p<0.05$. E: CD73 mRNA level of HPMEC treated with DHA for 36 and 48 hours. CD73 mRNA level is normalized to GAPDH mRNA level $(n=3)$, NS: No significant difference. F: Ineffectiveness of PPAR- $\gamma$ antagonist-GW9662 (10 $\mu \mathrm{mol} / \mathrm{l}, 72 \mathrm{~h}$ ) on CD73 protein expression quantified by Western blot $(n \geq 3)$.

Fig. 2. Effects of cholesterol reduction and supplementation on CD73 enzymatic activity in HPMEC (n=4-5). A: Treatment with filipin and B: MCD (10 mmol/l) and MCD-cholesterol-complex $\left(16 \mu \mathrm{g} / \mathrm{ml}, 30 \mathrm{~min}, 37^{\circ} \mathrm{C}\right)$. C: Effects of DHA and MCD on CD73 expression. D: Effects of DHA $(0,25$, $50 \mu \mathrm{mol} / \mathrm{l}$ ) on Cav-1 protein expression in HPMEC. Cav-1 protein was quantified by western blot and normalized to tubulin levels ( $\mathrm{n} \geq 3$ ).

these results MCD did not have a significant effect on CD73 expression stimulated with DHA (Fig. 2C). Hence, we concluded that membrane cholesterol content did not play a major role on regulation of CD73 expression in response to DHA.

In addition, the effect of DHA on cav-1 protein expression was examined (Fig. 2D). Although minor fluctuations of membrane Cav-1 expression were noted, these fluctuations appeared transient and no significant effect of DHA on cav-1 level could be objectified. Thus, increased expression of CD73 due to DHA treatment was not associated with changes of cav-1 expression. Similar results were observed with HUVEC (data not shown).
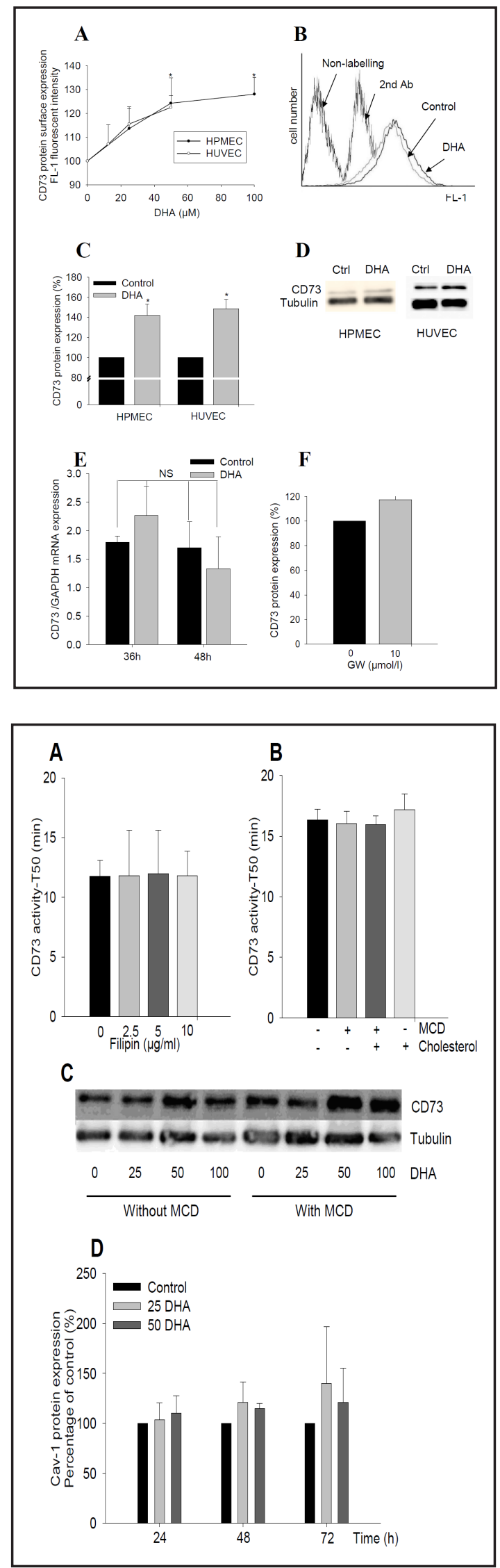
Fig. 3. A: Assessment of $\varepsilon$-AMP conversion to $\varepsilon$-Ado $(n=5), B$ : CD73 protein content $(n=9)$ of cell membrane and cytosolic fractions, respectively. Representative blots are numbered in accordance to columns. C: $\varepsilon$-AMP conversion to $\varepsilon$-Ado in intact HUVEC and HPMEC treated with $50 \mu \mathrm{mol} / \mathrm{l}$ DHA for $48 \mathrm{~h}(\mathrm{n}=6)$. D: same conditions as $\mathrm{C}$, however, in the absence and presence of levamisole $(50 \mu \mathrm{mol} / \mathrm{l})$ given $30 \mathrm{~min}$ before adding $\varepsilon$-AMP $(n=3)$. E: Extracellular $\varepsilon$-AMP conversion to EAdo in intact HUVEC under control conditions (no inhibitor), $50 \mu \mathrm{mol} / \mathrm{l}$ levamisole or/and 50 $\mu \mathrm{mol} / \mathrm{l}$ AOPCP for $30 \mathrm{~min}(\mathrm{n}=3)$. $t_{50}$ values represent time point of $50 \%$ conversion of $\varepsilon$-AMP. Values are given as percent change relative to $t_{50}$ of untreated controls. ${ }^{*} \mathrm{p}<0.05 ;{ }^{* *} \mathrm{p}<0.005{ }^{* * *} \mathrm{p}<0.001$ vs. untreated cells, \# $\mathrm{p}<0.001$ cytosolic versus membrane fraction, NS not significant.
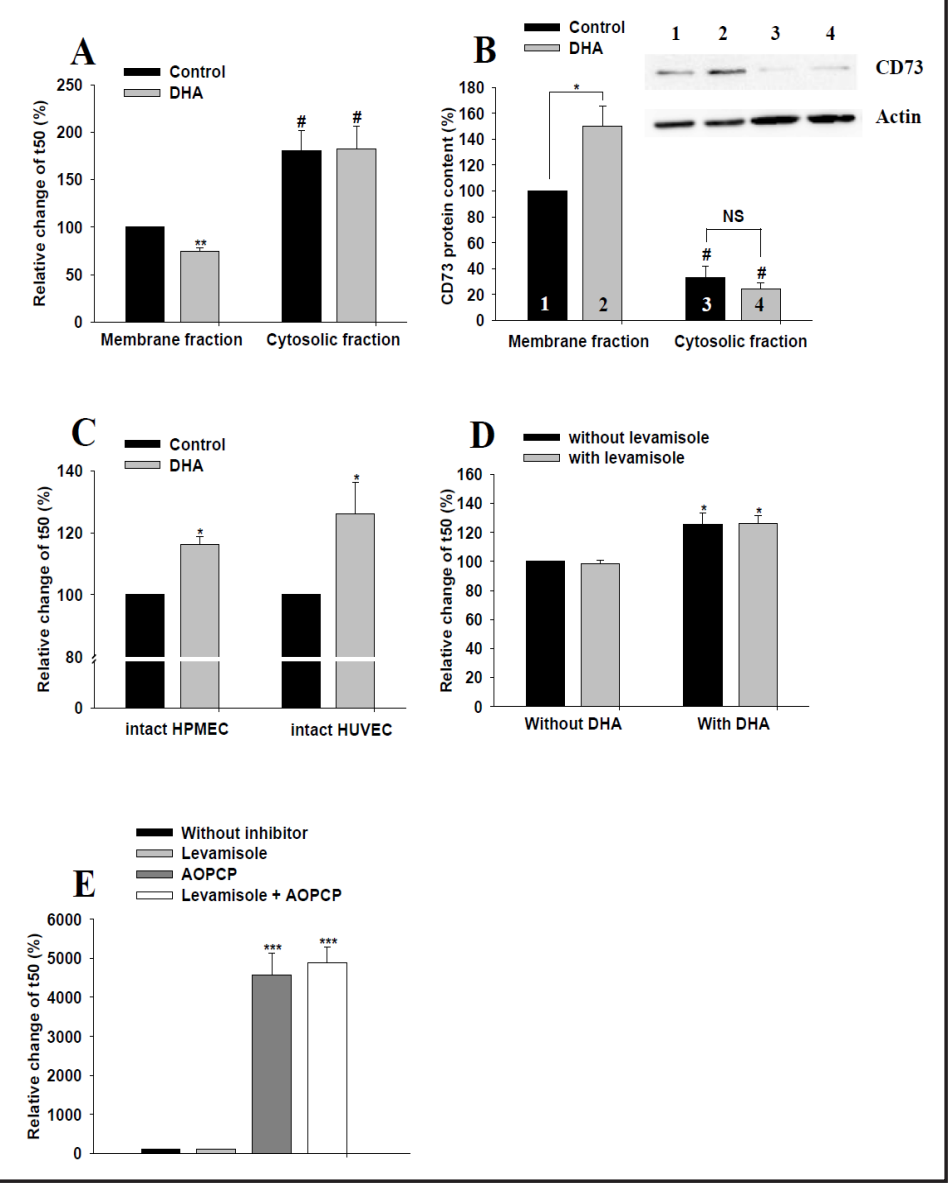

\section{Effects of DHA on CD73 enzymatic activity}

To test whether the change in CD73 expression results in a change of enzyme activity conversion of $\varepsilon$-AMP to $\varepsilon$-Ado was measured in both, membrane fraction and cytosolic fraction, after DHA treatment for $48 \mathrm{~h}$ (Fig. 3A). $\varepsilon$-AMP hydrolysis was about two-fold higher in the cell membrane fraction compared to the cytosolic fraction $(p<0.001)$. Upon DHA treatment $\varepsilon$-AMP hydrolysis was accelerated, indicated by decreased $t_{50}(25 \pm 4 \%)$. This effect was restricted to the enzyme activity of the membrane fraction $(\mathrm{p}<0.005)$. The results match with those obtained on CD73 protein content (Fig. 3B). CD73 protein content in the membrane fraction was 3-fold higher than that in the cytosolic fraction. DHA selectively elevated CD73 protein content by $50 \pm 15 \%$ in the membrane fraction. Thus, increased membrane CD73 protein content (Fig. 3B) was associated with increased enzyme activity (Fig. 3A).

Despite an enhanced membrane CD73 protein content in intact HUVEC and HPMEC DHA treatment resulted in prolonged $\varepsilon$-AMP degradation (increase of $t_{50} 16 \pm 2 \%$ and $26 \pm 10$ $\%$, Fig. 3C). Thus, conversion of extracellular $\varepsilon$-AMP to $\varepsilon$-Ado was slowed down on the whole cell level. Addition of the alkaline phosphatase inhibitor levamisole did not significantly affect hydrolysis rate of extracellular $\varepsilon$-AMP in response to DHA (Fig. 3D). In contrast, single inhibition of CD73 by its specific inhibitor alpha-beta-methylene-ADP (AOPCP) profoundly inhibited ع-AMP hydrolysis (Fig. 3E). Thus, in contrast to CD73 alkaline phosphatase is unimportant for AMP hydrolysis in isolated human endothelial cells.

Effects of DHA on CD39

Following DHA treatment ADPase (NTPDase1 or CD39) activity significantly decreased $(p<0.05)$ while ATPase activity remained unchanged (although there was a clear trend 
Fig. 4. Effects of DHA on CD39 activity (A) and protein level (B) in HUVEC. CD39 activity (A) was determined with $\varepsilon$-ADP and $\varepsilon$-ATP as substrates, respectively. CD39 protein expression (B) was analyzed by western blotting using mouse anti-human CD39 antibody, mean values $\pm \mathrm{SE}, \mathrm{n}=6$ experiments. C\&D: Effects of ARL-67156 on conversion of $\varepsilon$-ADP (C) and $\varepsilon$-ATP (D). E\&F: Effects of ARL-67156 and levamisole on conversion of $\varepsilon$-AMP to $\varepsilon$-Ado in HPMEC (E) and in HUVEC (F). Cells were treated with DHA for $48 \mathrm{~h}$. Inhibitors, ARL$67156(10 \mu \mathrm{mol} / \mathrm{l})$ and levamisole (50 $\mu \mathrm{mol} / \mathrm{l}$ ) were added $20 \mathrm{~min}$ before $\varepsilon$-AMP substrate. $\mathrm{t}_{50}$-Values are normalized to that of control cells treated in parallel. Mean values $\pm \mathrm{SE}, \mathrm{n}=3-6$ independent experiments, ${ }^{*} \mathrm{p}<0.05$ vs. untreated control and \# $\mathrm{p}<0.05$.

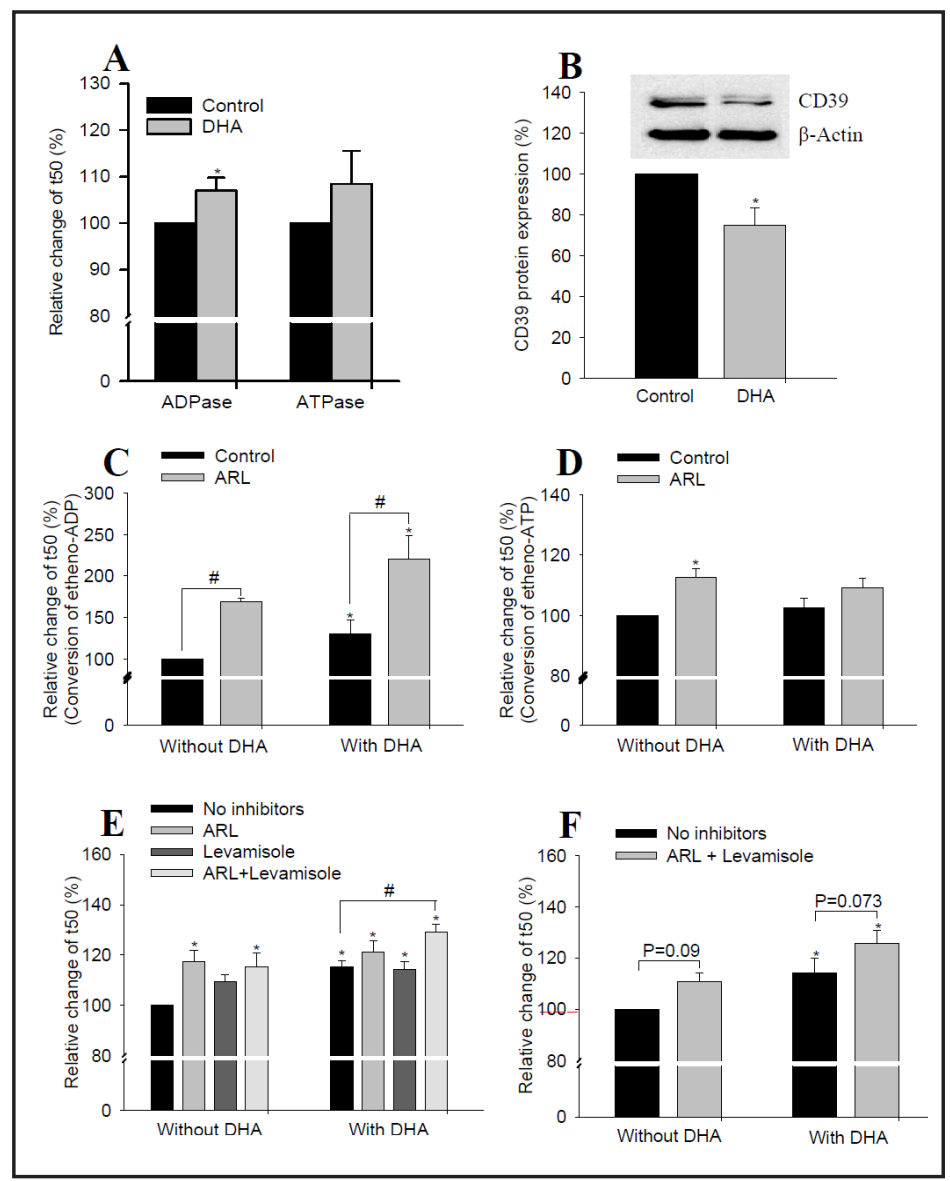

toward reduced activity, Fig. 4A). The decreased CD39 enzymatic activity was paralleled by a $25 \pm 8.5 \%$ decrease of CD39 protein expression ( $\mathrm{p}<0.05$, Fig. 4B). The functional importance of CD39 activity was addressed using the inhibitor ARL-67156 $(10 \mu \mathrm{mol} / \mathrm{l})$, which markedly decreased $\varepsilon$-ADP conversion (ADPase activity) in both DHA treated and DHA untreated groups (Fig. 4C). For the identical experimental conditions $\varepsilon$-ATP conversion rate (ATPase activity) showed a smaller effect (Fig. 4D).

Potential cross-talk between CD39, alkaline phosphatase and CD73 was studied using ARL-67156 as an inhibitor of CD39 and levamisole as an inhibitor of alkaline phosphatase. Single treatment with levamisole did not have any significant effect on extracellular $\varepsilon$-AMP hydrolysis in both, DHA untreated and DHA treated, HPMEC (Fig. 4E). In contrast, single treatment with ARL-67156 as well as combined treatment with ARL-67156 plus levamisole reduced extracellular $\varepsilon$-AMP conversion at baseline conditions (without DHA) as well as in presence of DHA (Fig. 4E). Similar results were observed in HUVEC in response to DHA treatment (Fig. 4F). This suggests that accumulation of extracellular ATP or ADP blunts CD73 activity in intact cells after DHA treatment.

\section{ATP release from endothelial cells treated with DHA}

Following treatment of HUVEC and HPMEC with DHA the ATP concentration in the supernatant was largely increased 5.5 \pm 0.9 -fold and 2.9 \pm 0.2 -fold, respectively (Fig. 5A and B). To assess that increased ATP release was not due to cell damage, we measured LDH release in parallel which did not change (data not shown). The different native adenine nucleotide fractions were assessed in the supernatant of HPMEC kept in incubation buffer for up to 32 min after a buffer change. The total extracellular nucleotide concentration increased 2.5-fold in the DHA treated group with respect to untreated HPMEC control (Fig. 5C). In addition, ATP and ADP levels of DHA treated cells remained elevated above those of control cells at 
Fig. 5. Effects of DHA on endothelial cell adenine nucleotide release. A: HUVEC, B: HPMEC. Cells were incubated in absence (control) or in presence of DHA for $48 \mathrm{~h}$. After change of cell buffer ATP release was determined by luminometry. Mean \pm SE normalized to control, $\mathrm{n} \geq 6$ experiments, ${ }^{* *} \mathrm{p}<0.01$ vs control. $\mathrm{C}$ : Fractions of adenine nucleotides released from HPMEC and measured with HPLC. ${ }^{*} \mathrm{p}<0.05$ refers to the sum of adenine compounds during control vs. DHA. D: Native AMP release from HPMEC. Data are taken from panel $\mathrm{C}$ and shown separately for better visualization. ${ }^{*} \mathrm{p}<0.05$ vs. time control, $\mathrm{n}=3$ independent experiments.
Fig. 6. Effects of ATP- $\gamma \mathrm{S}(\mathrm{A}), \mathrm{AOPCP}$ (B) on $\varepsilon$-AMP conversion to $\varepsilon$-Ado expressed as the relative change of $t_{50}$. HUVEC were incubated with ATP- $\gamma \mathrm{S}(15$ $\mathrm{min}$ ) or AOPCP (20 min) at concentrations indicated. $\varepsilon$-AMP $(5 \mu \mathrm{mol} / \mathrm{l})$ was added as substrate to assess CD73 enzymatic activity. Mean data \pm SE, $n \geq 3$ experiments, NS: not significant, ${ }^{*} \mathrm{p}<0.05$; ${ }^{* *} \mathrm{p}<0.005 ;{ }^{* * *} \mathrm{p}<0.0001$ vs. control.
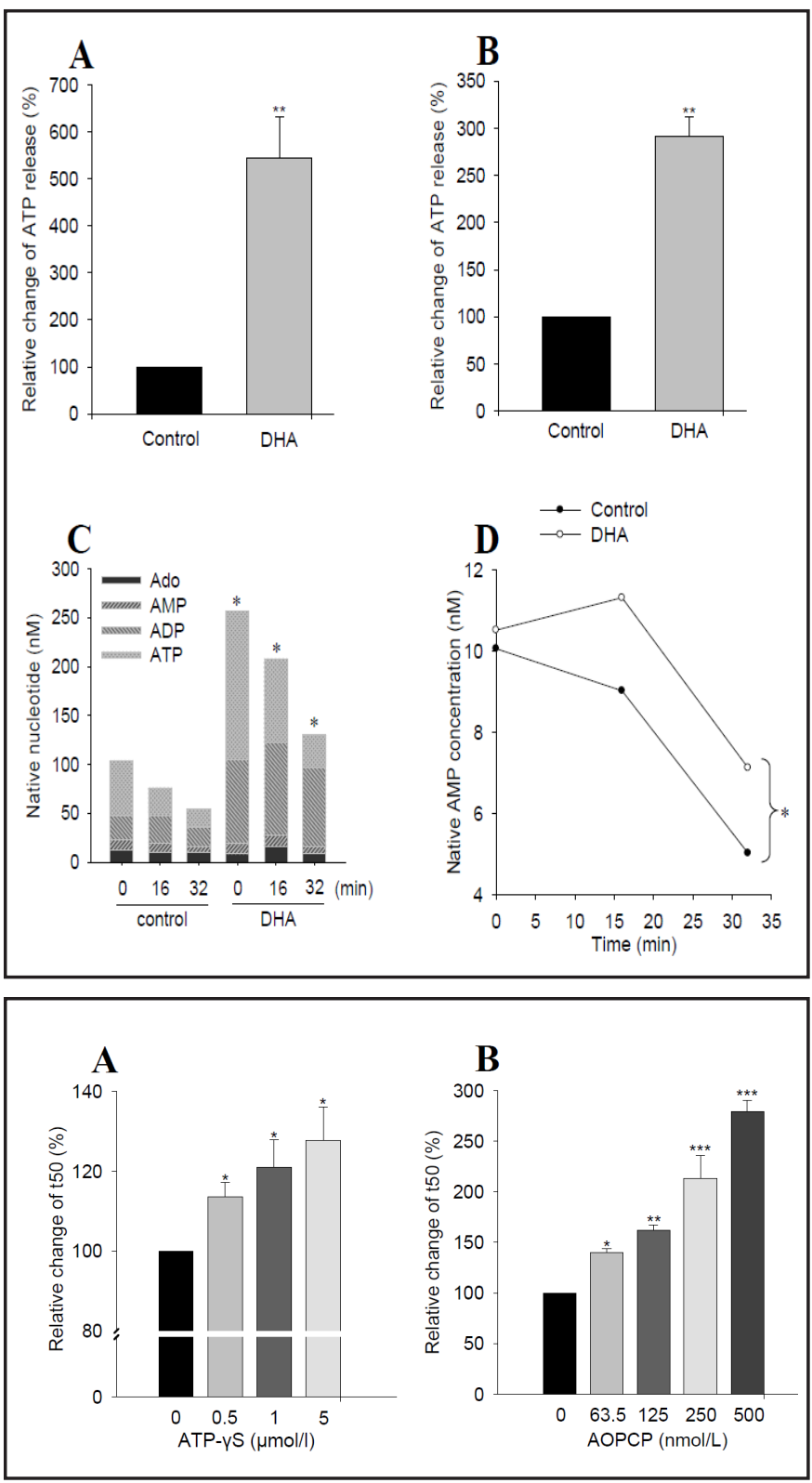

all time points $(\mathrm{p}<0.05)$. Without CD39 inhibition, extracellular ATP concentration declined as a function of time in both control and DHA treated groups. Interestingly, ADP levels (27 $\mathrm{nmol} / \mathrm{l}$ in the control group and $94 \mathrm{nmol} / \mathrm{l}$ in the DHA treated group) remained unchanged over $16 \mathrm{~min}$ and even thereafter the decline was small. In parallel, the conversion of AMP to Ado was decreased in the DHA treated group, but maintained in the control group (Fig. 5D). Thus, ATP is released from DHA-stimulated human endothelial cells at an increased rate and its metabolism via ecto-nucleotidase(s) is diminished.

\section{Inhibition of CD73 by ATP- $\gamma S$ and $A O P C P$}

The effects of nucleotide analogues on CD73 activity for our experimental conditions are summarized in Fig. 6. The stable ATP analogue ATP- $\gamma$ S dose-dependently inhibited $\varepsilon$-AMP conversion rate by about $14 \pm 4 \%$ at $500 \mathrm{nmol} / \mathrm{l}$ and $28 \pm 8 \%$ at $5 \mu \mathrm{mol} / \mathrm{l}$ concentration in comparison to control ( $\mathrm{p}<0.05$, Fig. 6A). A similar result was observed in HPMEC (data not shown). An even stronger inhibitory effect was obtained for the stable ADP analogue AOPCP, which increased $t_{50}$ by $40 \pm 4 \%$ to $180 \pm 11 \%$ at AOPCP concentrations ranging from 63.5 to 
$500 \mathrm{nmol} / \mathrm{l}(\mathrm{p}<0.05$, Fig. 6B). Thus, CD73 activity is highly sensitive to inhibition by ATP- and ADP-analogues in human endothelial cells.

\section{Discussion}

The present study demonstrates that DHA exposure of human endothelial cells results in a complex reorganization of extracellular adenine nucleotide metabolism including 1) increased CD73 protein content, 2) decreased CD39 expression associated with decreased catalytic activity, and 3) enhanced cellular adenine nucleotide release. These effects interact on the whole cell level by augmenting extracellular adenine nucleotide concentrations and consecutively decreasing CD73 activity despite an increased CD73 expression. Thus, DHA increases the extracellular adenine nucleotide/adenosine ratio.

After $48 \mathrm{~h}$ DHA treatment CD73 surface expression and protein level were increased in human endothelial cells (Fig. 1). This is in line with a previous study performed in rat endothelial cells [7]. Moreover, our study determines that the increased CD73 activity is strictly related to cell surface expression (Fig. 1A, 1B). Several mechanisms of CD73 protein content regulation have been described in literature including modulation of plasma membrane fluidity [7], modulation of lipid raft association with caveolin-1 [22], regulation of transcription processes [23], and cholesterol-dependent endocytosis [24]. We have tested whether these concepts apply to the conditions of the current study using RT-PCR to assess the mRNA level and transcriptional regulation of CD73. However, no change in CD73 mRNA level was found (Fig. 1E). Because DHA was shown to be a ligand for PPAR- $\gamma$ [25] which may regulate CD73 transcription processes, the PPAR- $\gamma$ antagonist GW9662 was applied. In line with the negative RT-PCR result, GW9662 had no effect on CD73 protein level (Fig. 1F). Furthermore, modification of plasma membrane cholesterol either by depletion of cholesterol using filipin and $\beta$-methyl-cyclodextrin (MCD) or by enrichment using cholesterol-MCD complex had no impact on CD73 enzyme activity and protein level (Fig. 2A-C). Also, cav-1 protein level was unaffected by DHA treatment (Fig. 2D). It is thus unlikely that previously proposed mechanisms were effective under the conditions of our study.

One previous study performed in isolated cell membrane preparations indicated increased CD73 expression and enzyme activity after DHA treatment [7]. Our study clearly shows that this view needs correction. While our experiments confirm results obtained on the membrane level (Fig. s 1-3), the new and important finding is that DHA reduced CD73 activity in intact endothelial cells (Fig. 3). This is in contrast to the conclusion based on experiments using membrane preparations [7]. The present study reveals that the effect of enhanced membrane expression of CD73 in response to DHA is completely set-off by (an) other mechanism(s) on the level of the intact cell.

Ecto-nucleotidases are regulated by a variety of factors including adenine nucleotides. In fact, CD73 is powerfully inhibited by ADP and ATP [16, 26] (Fig. 6A, B). As shown in Fig. 5 , DHA treatment largely enhanced adenine nucleotide release. The increased extracellular ATP and ADP concentrations blunted CD73 activity under the conditions of the present study (Fig. 4E, F). We therefore conclude that the increase of CD73 expression on the cell membrane level was counteracted by augmented ATP release from endothelial cells und subsequent block of its hydrolysis. In endothelial cells ATP release has been suggested to occur via P-glycoprotein, CFTR, connexin hemichannels or pannexins [27-29] although their quantitative contribution is currently unknown.

HUVEC released ATP under control conditions and after DHA treatment at a rate resulting in a nanomolar adenine nucleotide concentration in the cell supernatant. Most notably, the adenine nucleotide fraction was largely elevated in the DHA treated vs. untreated group. While extracellular ATP continuously declined, native ADP level appeared rather constant in the course of the experiment (Fig. 5C). Although the adenosine level slightly increased over time in the DHA group, it should be stressed that the adenosine fraction was always considerably smaller than those of the adenine nucleotides, in particular the ATP- and ADPfractions. 
Fig. 7. Schematic diagram showing the effects of DHA on regulation of extracellular adenine nucleotide metabolism in human endothelial cells. e-NTP: ecto-nucleoside-triphosphatepyrophosphatase.

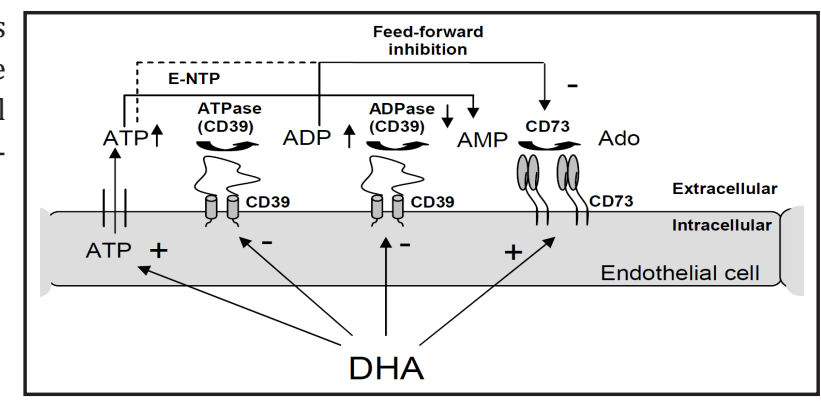

The metabolic processing of extracellular adenine nucleotides includes a complex cascade of various ecto-enzymes on the cell surface [4, 30, 31]. Key enzymes expressed on human endothelial cells for forward reactions from ATP to ADP to AMP and to adenosine include CD39 and CD73. Backward reactions from AMP to ADP and ATP are catalyzed by ecto-kinases, e.g. adenylate kinase, NDPkinase, ATP synthase. Simultaneous release of ATP and other nucleotides such as ADP and AMP cause additive conformational change of ectokinases pushing the backward reaction to produce more extracellular ATP and ADP. Changes in the ratio of mono-, di- and tri-phosphates may cause feed-forward inhibition of CD73 by the precursors ATP and ADP, respectively [27, 30, 31]. The $K_{i}$ values reported for inhibition of CD73 by ATP are in the low micromolar range and that for inhibition by ADP in the low nanomolar range $[15,16,32]$. The concentrations of ATP and ADP measured in response to DHA treatment were in this range $(100-200 \mathrm{nmol} / \mathrm{l})$, while those measured under control conditions were lower (20-50 nmol/l). Thus, quantitative assessment of ATP and ADP concentrations after DHA treatment indicate a role as autocrine inhibitors of CD73 activity in human endothelial cells.

In HPMEC and HUVEC inhibition of CD39 by ARL-67156 resulted in a decrease of CD73 activity, both under control conditions and after DHA treatment (Fig. 4E, F). This supports the concept that native extracellular adenine nucleotide concentrations regulate activity of CD73. Because CD73 catalytic activity was inhibited following application of ARL-67165 under baseline conditions as well as after DHA treatment, the resulting extracellular ATP or ADP concentrations were likely effective in modulating CD73 activity under the conditions of our study. This effect is supported by a down-regulation of CD39 protein expression and ecto-ADPase enzymatic activity following DHA (Fig. 4A, B). The results obtained in our reductionist model may help to clarify the mechanisms that mediate the beneficial clinical effects seen after DHA treatment $[8,9,33]$. However, it needs also to be taken into account that the in vivo situation is more complex with e.g. parenchymal cells, platelets, leukocytes and red blood cells representing additional major sources of cellular ATP release. A beneficial effect of an increased adenine nucleotide/adenosine ratio is compatible with an improved endothelial barrier function following block of CD73 [3]. However, it should also be pointed out that a blunted capability of adenosine production by CD73 may be detrimental under conditions of acute lung injury induced by mechanical ventilation [4] or under hypoxic stress in lung [5] or intestinal mucosa [6]. Thus, it is unlikely that the beneficial effects of DHA treatment are caused by adenosine, because its production is rather decreased after DHA (Fig. 3).

Figure 7 summarizes our results. DHA up-regulated CD73 protein level as well as CD73 surface expression. However, CD73 enzymatic activity of human endothelial cells was diminished via feed-forward inhibition by augmented cellular release of ADP and ATP despite increased CD73 surface expression. Because DHA reduced CD39 activity and expression and increased ATP release the extracellular ATP/adenosine ratio was augmented. Because both metabolites act on separate receptors and, hence, separate signal transduction pathways, this concerted action may exert a profound regulatory role. The current study shows that for a comprehensive evaluation of effects seen after DHA substitution the complex regulation of the extracellular adenine nucleotide/ adenosine metabolism must be taken into account rather than single assessment of CD73 expression and activity. 
Thom/Wendel/Deussen: DHA and Extracellular Adenine Nucleotide Metabolism

\section{Acknowledgements}

We gratefully acknowledge the support with HPMEC cells (HPMEC-ST1.6R) from Prof. Dr. Kirkpatrick, Institute of Pathology, Johannes-Gutenberg-Universität Mainz, Germany. We acknowledge the expert technical assistance of Sandra Tuchscheerer-Hoffmeister and Bianca Müller in cell culture and sample analysis. The current address of Dr. Martina Wendel is Zentrum für Psychiatrie Südwürttemberg, Department of Child and Adolescent Psychiatry, Weingartshofer Str. 2D-88214 Ravensburg, Germany and that of Dr. Vu Thi Thom is School of Medicine and Pharmacy, Vietnam National University, Vietnam.

This work was supported by a grant from the Bundesministerium für Bildung and Forschung (BMBF) for the PhD program "Endothelium and Metabolism" of the Medical Faculty Carl Gustav Carus at TU Dresden, the International PhD-Program "Endothelium in Health and Disease" funded by BMBF within the IPID-program executed by DAAD and a grant given by Vietnam Ministry of education and training.

\section{Conflict of Interest}

The authors declare no conflicts of interest, financial or otherwise.

\section{Refrences}

1 Schreiber R, Castrop H, Kunzelmann K: Allergen-induced airway hyperresponsiveness is absent in ecto-5'nucleotidase (CD73)-deficient mice. Pflügers Arch 2008;457:431-440.

- Peng Z, Fernandez P, Wilder T, Yee H, Chiriboga L, Chan ESL, Cronstein BN: Ecto-5'-nucleotidase (CD73)mediated extracellular adenosine production plays a critical role in hepatic fibrosis. Faseb J 2008;22:22632272.

- G Gündüz D, Hirche F, Härtel FV, Rodewald CW, Schäfer M, Pfitzer G, Piper HM, Noll T: ATP antagonism of thrombin-induced endothelial barrier permeability. Cardiovasc Res 2003;59:470-478.

-4 Eckle T, Füllbier L, Wehrmann M, Khoury J, Mittelbronn M, Ibla J, Rosenberger P, Eltzschig HK: Identification of ectonucleotidase CD39 and CD73 in innate protection during acute lung injury. J Immunol 2007;178:8127-8137.

5 Thompson LF, Eltzschig HK, Ibla JC, Van De Wiele CJ, Resta R, Morote-Garcia JC, Colgan SP: Crucial role for Ecto-5'-nucleotidase (CD73) in vascular leakage during hypoxia. J Exp Med 2004;200:1395-1405.

-6 Synnestvedt K, Furuta GT, Comerford KM, Louis N, Karhausen J, Eltzschig HK, Hansen KR, Thompson LF, Colgan SP: Ecto-5'-nucleotidase (CD73) regulation by hypoxia-inducible factor-1 mediates permeability changes in intestinal epithelia. J Clin Invest 2002;110:993-1002.

7 Hashimoto M, Hossain S, Tanabe Y, Shido O: Effects of aging on the relation of adenyl purine release with plasma membrane fluidity of arterial endothelial cells. Prost Leuk Ess Fat Acids 2005;73:475-483.

8 Heller AR, Koch T: Role of omega-3 FA in acute lung injury; in Heller AR, Stehr SN, Koch T (eds.): Omega 3 fatty acids in clinical nutrition. NOVA Publishers, 2006, pp 17-23.

-9 Gadeck JE, DeMichele SJ, Karlstad MD, Pacht ER, Donahoe M, Albertson TE, van Hoozen C, Wennberg AK, Nelson JL, Noursalehi M: Effect of enteral feeding with eicosapentaenoic acid, gamma-linolenic acid, and antioxidants in patients with acute respiratory distress syndrome. Enteral Nutrition in ARDS Study Group. Crit Care Med 1999;27:1409-1420

10 Weber C, Erl W, Pietsch A, Danesch U, Weber PC: Docosahexaenoic acid selectively attentuates induction of vascular cell adhesion molecule- 1 and subsequent monocytic cell adhesion to human endothelial cells stimulated by tumor necrosis factor- $\alpha$. Art Thromb Vasc Biol 1995;15:622-628.

11 Zernecke A, Bidzhekov K, Özüyaman B, Fraemohs L, Liehn EA, Lüscher-Firzlaff JM, Lüscher B, Schrader J, Weber C: CD73/Ecto-5'-nucleotidase protects against vascular inflammation and neointima formation. Circulation 2006;113:2120-2127. 


\section{Cellular Physiology Cell Physiol Biochem 2013;32:355-366 and Biochemistry

12 Wilson HL, Varcoe RW, Stokes L, Holland KL, Francis SE, Dower SK, Surprenant A, Crossman DC: P2X receptor characterization and IL-1/IL-1Ra release from human endothelial cells. Br J Pharmacol 2007;151:96-108.

13 Kawai Y, Yokoyama Y, Kaidoh M, Ohhashi T: Shear stress-induced ATP-mediated endothelial constitutive nitric oxide synthase expression in human lymphatic endothelial cells. Am J Physiol 2009;98:C647-C655.

14 Corriden R, Insel PA: Basal release of ATP: An autocrine-paracrine mechanism for cell regulation. Sci Signal 2010;3: re1.

15 Burger RM, Lowenstein JM: 5'-Nucleotidase from smooth muscle of small intestine and from brain. Inhibition by nucleotides. Biochemistry 1975;14:2362-2366.

16 Naito Y, Lowenstein JM: 5'-Nucleotidase from rat heart membranes. Inhibition by adenine nucleotides and related compounds. J Biochem 1985;226:645-651.

17 Andrade CM, Roesch GC, Wink MR, Guimaraes EL, Souza LF, Jardim FR, Guaragna RM, Bernard EA, Margis R, Borojevic R, Battastini AM, Guma FC: Activity and expression of ecto-5'-nucleotidase/CD73 are increased during phenotype conversion of a hepatic stellate cell line. Life Sci 2008;82:21-29.

18 Krump-Konvalinkova V, Bittinger F, Unger RE, Peters K, Lehr HA, Kirkpatrick CJ: Generation of human pulmonary microvascular endothelial cell lines. Lab Invest 2001;81:1717-1727.

19 Pexa A, Deussen A: Modulation of ecto-5'-nucleotidase by phospholipids in human umbilical vein endothelial cells (HUVEC). Naunyn-Schmiedeberg's Arch Pharmacol 2005;372:131-138.

20 Haink G, Deussen A: Liquid chromatography method for the analysis of adenosine compounds. J Chromatogr B Analyt Technol Biomed Life Sci 2003:784:189-193.

-21 Larsson SC, Kumlin M, Ingelman-Sundberg M, Wolk A: Dietary long-chain n-3 fatty acids for the prevention of cancer: a review of potential mechanism. Am J Clin Nutr 2004;79:935-945.

-22 Strohmeier GR, Lencer WI, Patapoff TW, Thompson LF, Carison SL, Moe SJ, Carnes DK, Mrsny RJ, Madara JL: Surface expression, polarization, and functional significance of CD73 in human intestinal epithelia. J Clin Invest 1997;99:2588-2601.

23 Niemelae J, Henttinen T, Yegutkin GG, Airas L, Kujari AM, Rajala P, Jalkanen S: IFN- $\alpha$ induced adenosine production on the Endothelium: A mechanism mediated by CD73 (Ecto-5'-nucleotidase) up-regulation. J Immunol 2004;172:1646-1653.

24 Ledoux S, Laouari D, Essig M, Runembert, Trugnan G, Michel JB, Friedlander G: Lovastatin enhances ecto5 '-nucleotidase activity and cell surface expression in endothelial cells. Implication of Rho-family GTPase. Circ Res 2002;90:420-427.

-25 Lee SA, Kim HJ, Chang KC, Baek JC, Park JK, Shin JK, Choi WJ, Lee JH, Paik WY: DHA and EPA down-regulate COX-2 expression through suppression of NF- $\mathrm{KB}$ activity in LPS-treated human umbilical vein endothelial cells. Korean J Physiol Pharmacol 2009;13:301-307.

26 Zimmermann H: 5'-Nucleotidase: molecular structure and functional aspects. J Biochem 1992;285:345365.

27 Yegutkin GG: Nucleotide- and nucleoside-converting ectoenzymes: Important modulators of purinergic signaling cascade. Biochim Biophys Act 2008;1783:673-694.

-28 Gödecke S, Roderigo C, Rose CR, Rauch BH, Gödecke A, Schrader J: Thrombin-induced ATP release from human umbilical vein endothelial cells. Am J Physiol Cell Physiol 2012;302:C915-C923.

-29 Lohman AW, Billaud M, Straub AC, Johnstone SR, Best AK, Lee M, Barr K, Penuela S, Laird DW, Isakson BE: Expression of pannexin isoforms in the systemic murine arterial network. J Vasc Res 2012;49:405-416.

-30 Yegutkin GG, Henttinen T, Samburski SS, Spychala J, Jalkanen S: The evidence for two opposite, ATPgenerating and ATP-consuming, extracellular pathways on endothelial and lymphoid cells. J Biochem 2002;367:121-128.

-31 Yegutkin GG, Henttinen T, Jalkanen S: Extracellular ATP formation on vascular endothelial cells is mediated by ecto-nucleotide kinase activities via phosphotransfer reactions. FASEB J 2001;15:251-260.

-32 Sullivan JM, Alpers ZB: In vitro regulation of rat heart 5'-nucleotidase by adenine nucleotides and magnesium. J Biol Chem 1971;246:3057-3063.

-33 Marcus AJ, Broekman MJ, Drosopoulos JHF, Levi R: Role of CD39 (NTPDase-1) in thromboregulation, cerebroprotection, and cardioprotection. Sem Thromb Hem 2005;31:234-246. 